\title{
Stability of two-dimensional complex plasma monolayers in asymmetric capacitively coupled radio-frequency discharges
}

\author{
L. Couëdel $\odot^{1,2, *}$ and V. Nosenko $\odot^{3}$ \\ ${ }^{1}$ Physics and Engineering Physics Department, University of Saskatchewan, Saskatoon, Saskatchewan S7N 5E2, Canada \\ ${ }^{2}$ CNRS, Aix-Marseille Université, Laboratoire PIIM UMR 7345, 13397 Marseille cedex 20, France \\ ${ }^{3}$ Institut für Materialphysik im Weltraum, Deutsches Zentrum für Luft- und Raumfahrt (DLR), D-82234 Weßling, Germany
}

(Received 14 October 2021; accepted 11 January 2022; published 28 January 2022)

\begin{abstract}
In this article, the stability of a complex plasma monolayer levitating in the sheath of the powered electrode of an asymmetric capacitively coupled radio-frequency argon discharge is studied. Compared to earlier studies, a better integration of the experimental results and theory is achieved by operating with actual experimental control parameters such as the gas pressure and the discharge power. It is shown that for a given microparticle monolayer at a fixed discharge power there exist two threshold pressures: (i) above a specific pressure $p_{\text {cryst }}$, the monolayer always crystallizes; (ii) below a specific pressure $p_{\mathrm{MCI}}$, the crystalline monolayer undergoes the mode-coupling instability and the two-dimensional complex plasma crystal melts. In between $p_{\mathrm{MCI}}$ and $p_{\text {cryst }}$, the microparticle monolayer can be either in the fluid phase or the crystal phase: when increasing the pressure from below $p_{\mathrm{MCI}}$, the monolayer remains in the fluid phase until it reaches $p_{\text {cryst }}$ at which it recrystallizes; when decreasing the pressure from above $p_{\text {cryst }}$, the monolayer remains in the crystalline phase until it reaches $p_{\mathrm{MCI}}$ at which the mode-coupling instability is triggered and the crystal melts. A simple self-consistent sheath model is used to calculate the rf sheath profile, the microparticle charges, and the microparticle resonance frequency as a function of power and background argon pressure. Combined with calculation of the lattice modes the main trends of $p_{\mathrm{MCI}}$ as a function of power and background argon pressure are recovered. The threshold of the mode-coupling instability in the crystalline phase is dominated by the crossing of the longitudinal in-plane lattice mode and the out-of plane lattice mode induced by the change of the sheath profile. Ion wakes are shown to have a significant effect too.
\end{abstract}

DOI: 10.1103/PhysRevE.105.015210

\section{INTRODUCTION}

Two-dimensional (2D) complex plasma crystals are composed of negatively charged monosized spherical microparticles levitating in the sheath above a confining electrode [1]. Complex plasma crystals are generally studied in asymmetric capacitively coupled radio-frequency (cc-rf) argon discharges in which the injected monolayer of microparticles levitates in the sheath above the powered electrode and crystallizes under specific discharge conditions [2-9]. Since microparticles can easily be imaged thanks to laser light scattering and the use of fast cameras, microparticle trajectories can be recovered and one can obtain information about the crystal at the kinetic "particle" level. Thus, complex plasma crystals often serve as model systems to study generic phenomena such as wave propagation $[5,10]$, shocks $[11,12]$, and phase transitions [13-16].

However, due to the very nature of the complex plasma monolayer, complex plasma specific phenomena can occur. Because of the ion flow coming from the bulk plasma and directed toward the electrode, ion wakes are formed downstream of each microparticle [4,17-21]. The ion wakes exert attractive force on the neighboring particles making the

\footnotetext{
*lenaic.couedel@usask.ca; lenaic.couedel@univ-amu.fr
}

microparticle-microparticle interactions nonreciprocal. Under specific conditions, these nonreciprocal interactions can trigger the mode-coupling instability (MCI) $[9,22-26]$ in which energy from the flowing ions is transferred to the microparticles and can heat up the microparticle suspension. Indeed, in 2D complex plasma crystals, three wave modes can be sustained: longitudinal and transverse in-plane acoustic modes and, due to the finite strength of the vertical confinement, an out-of-plane optical mode (associated with vertical oscillations $[7,22,24,27,28])$. Due to wake attraction, when the longitudinal in-plane mode and the out-of-plane mode cross, an unstable hybrid mode is formed (the typical fingerprints being a hot spot in reciprocal space at the edge of the first Brillouin zone, angular dependence, and mixed polarization [24,25]), which can trigger MCI if the instability growth rate is larger than the damping rate due to friction with the neutral background [24]. MCI is observed in both crystalline $[9,24,25]$ and fluid monolayers [26,29]. In the first case, MCI can result in rapid melting of the monolayer if its growth rate is high enough. In the latter case, MCI can prevent crystallization of the monolayer by pumping more kinetic energy into the microparticles than the energy dissipated through friction with the neutral gas background [29-31]. Since for given discharge parameters (rf power, neutral gas pressure, geometry) and fixed monolayer parameters (number density and size of the microparticles) the growth rate in a fluid monolayer is 
higher than in a crystalline monolayer [26], it allows external triggering of the MCI [32] and prevents recrystallization of a melted monolayer without strongly altering the discharge parameters [29,32].

It was shown experimentally that MCI is triggered at specific pressure and rf power thresholds [9,25]. MCI is very sensitive to the magnitude of the effective wake dipole moment since the shape of the dispersion relations critically depends on it [33]. MCI threshold and growth rate also strongly depend on the depth of the mode crossing [33], which is a function of the ion-wake parameters and vertical confinement frequency. Both the ion wake and the vertical confinement frequency depend on the parameters of the rf sheath in which the microparticles are levitating such as the strength of the confining sheath electric field and the local ion and electron densities. Therefore, in order to understand the dynamics of the microparticle monolayer, it is very important to be able to relate the discharge parameters (background argon pressure, rf power) to the sheath parameters (electric field profile, ion flux profile, ion and electron density profiles). Modeling the rf sheath in a cc-rf discharge is however not trivial and accurate calculation of the rf sheath profile has been a subject of active research for the past three decades; see for example Refs. [34-43].

In this article, the stability of $2 \mathrm{D}$ complex plasma monolayers levitating in the sheath above the powered electrode of a cc-rf discharge is experimentally studied. In Sec. II, the experimental setup is described. In Sec. III, the experimental characterization of the cc-rf discharge (electron density and powered electrode self-bias) as a function of the background argon pressure and the forward rf power is presented. In Sec. IV, a study of the stability (crystal or fluid) of specific microparticle monolayers as a function of rf power is performed. It allows us to measure the MCI pressure threshold and the crystallization pressure threshold over a wide range of rf powers. In Sec. V, a simple self-consistent sheath model is described and used to calculate the sheath profile and microparticle charge, and vertical resonance frequency as a function of discharge parameters. In Sec. VI, the results of Sec. V are used to calculate the lattice modes of 2D complex plasma crystal with and without ion wakes. The influence of the interparticle distance and wake parameters are investigated. The calculated results are compared to experimental results. In Sec. VII, the main results are summarized and future investigations are discussed.

\section{EXPERIMENTAL SETUP}

The experiments were performed in a modified gaseous electronic conference rf cell (GEC). It is an asymmetric cc-rf discharge at $13.56 \mathrm{MHz}$. A sketch of the setup is shown in Fig. 1. Experiments were performed with an argon pressure, $p_{\text {Ar }}$, between 0.5 and $2 \mathrm{~Pa}$. The forward rf power, $P_{\mathrm{W}}$, was set between 5 and $25 \mathrm{~W}$. Langmuir probe studies using the same setup have shown that the electron temperature is $T_{e} \sim$ $2.5 \mathrm{eV}$ and that the electron density is $n_{e} \simeq 2 \times 10^{9} \mathrm{~cm}^{-3}$ at $p_{\mathrm{Ar}}=0.66 \mathrm{~Pa}$ and $P_{\mathrm{W}}=20 \mathrm{~W}$ [44]. A Miwitron Ka-band microwave interferometer MWI 2650 working at $26.5 \mathrm{GHz}$ was used to measure the electron density, $n_{e}$, in pristine plasma

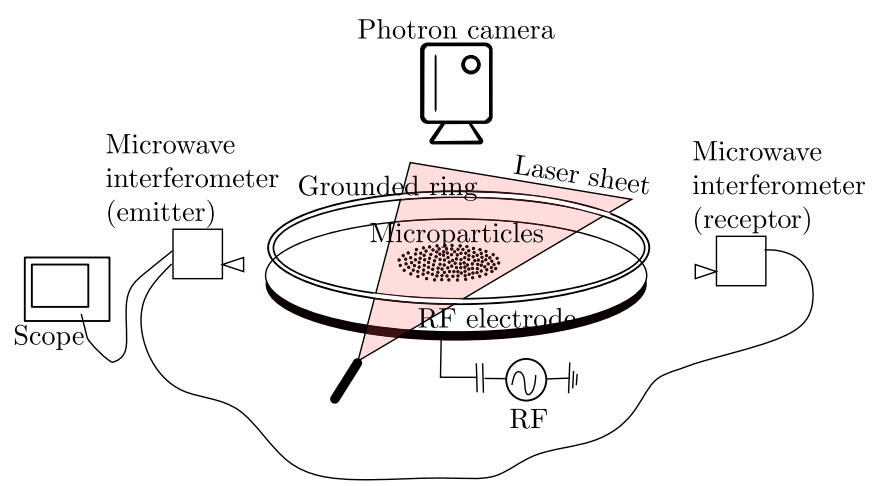

FIG. 1. Schematic of the experimental setup. Note that the microwave interferometer was used only for measurements in pristine plasmas (i.e., no microparticles).

condition (without injected microparticles) for different $p_{\mathrm{Ar}}$ and $P_{\mathrm{W}}$. The interferometer had a resolution of $\sim 10^{8} \mathrm{~cm}^{-3}$.

Melamine-formaldehyde (MF) spherical microparticles with a diameter of $9.19 \pm 0.09 \mu \mathrm{m}$ were injected in the plasma. They levitated in the sheath above the lower rf electrode where the electric force balanced their weight and formed a highly ordered horizontal monolayer. The microparticle monolayer was illuminated by a horizontal laser sheet and the microparticles were imaged through the top chamber window by using a 4 megapixel Photron FASTCAM Mini WX100 camera at a speed of 250 frames per second. Particle tracking allowed us to recover the particle horizontal coordinates, $x$ and $y$, with subpixel resolution in each frame, and the velocities, $v_{x}$ and $v_{y}$, were then calculated [8]. An additional side-view camera (Basler Ace ACA640-100GM) was used to check that no particles were levitating above or under the main monolayer. More details can be found in previous publications [9,44-46].

\section{CHARACTERIZATION OF THE CC-RF DISCHARGE}

Electron density measurements have been taken with the microwave interferometer for different pressure between $0.66 \mathrm{~Pa}$ and 6.0 $\mathrm{Pa}$ and for rf power between $1 \mathrm{~W}$ and $20 \mathrm{~W}$. The results are are shown in Fig. 2. The density reported at $p_{\mathrm{Ar}}=0.66 \mathrm{~Pa}$ and $P_{\mathrm{W}}=20 \mathrm{~W}$ is $n_{e} \sim 2.5 \times 10^{9} \mathrm{~cm}^{-3}$ which is in agreement with previous Langmuir probe measurements [44]. As expected, $n_{e}$ increases with forward $\mathrm{rf}$ power and pressure $[41,47]$. The measured values of bulk plasma density are used as input parameters for sheath profile calculations in Sec. V C.

The absolute value of self-bias voltage on the powered electrode, $\left|V_{\mathrm{dc}}\right|$, has also been measured for a few relevant experimental discharge conditions. The results are shown in Fig. 3 (dots). As can be seen, $\left|V_{\mathrm{dc}}\right|$ is not very sensitive to pressure. It has approximately a square-root dependence on the forward $\mathrm{rf}$ power.

It is known that the self-bias voltage is related to the ratio of the areas of the powered surface to the grounded surface interacting with the plasma $A_{\mathrm{rf}} / A_{g}[47,48]$. The ratio of the self-bias voltage $V_{\mathrm{dc}}$ to the amplitude of the rf voltage 


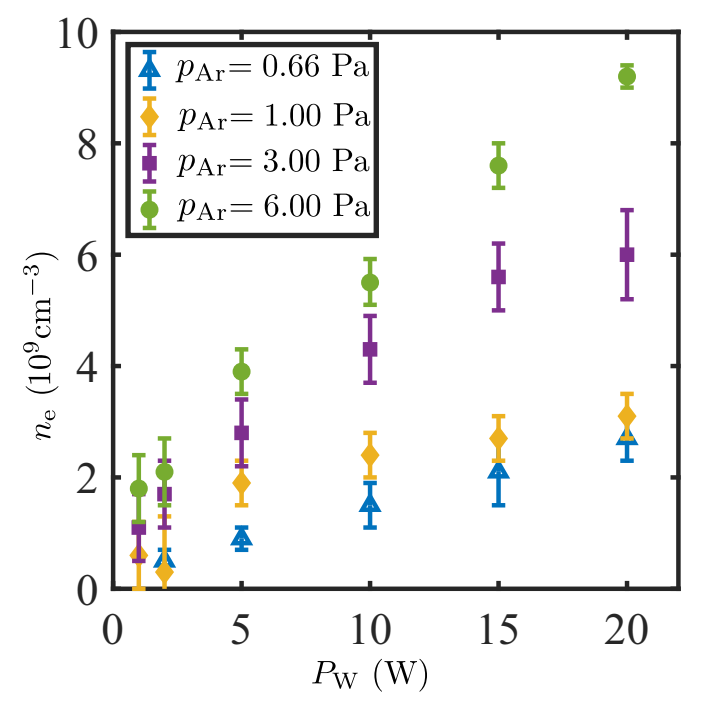

FIG. 2. Electron density, $n_{e}$, as a function of rf power, $P_{\mathrm{W}}$, for different pressures.

$V_{0}=V_{\mathrm{pp}} / 2$ is in first approximation given by $[49,50]$

$$
\frac{V_{\mathrm{dc}}}{V_{0}}=\sin \left[\frac{\pi}{2}\left(\frac{A_{g}-A_{\mathrm{rf}}}{A_{g}+A_{\mathrm{rf}}}\right)\right],
$$

where $V_{\mathrm{pp}}$ is the peak-to-peak rf voltage. It was experimentally measured that in a modified GEC cell similar to the one that was used for the current set of experiments, $A_{\mathrm{rf}} / A_{g} \sim 0.25$ [50]. Using the fit of $V_{\mathrm{dc}}$ as a function of $P_{\mathrm{W}}$ and Eq. (1), $V_{\mathrm{pp}}$ was calculated and the result is shown by the dotted red line in Fig. 3. The results match reasonably well with two measured values of $V_{\mathrm{pp}}$ at $15 \mathrm{~W}$ and $20 \mathrm{~W}$ for $p=0.66 \mathrm{~Pa}$ (blue squares). The calculated values of $V_{\mathrm{dc}}$ and $V_{\mathrm{pp}}$ are used as input parameters to calculate sheath profiles in Sec. V C.

\section{STABILITY OF COMPLEX PLASMA MONOLAYERS}

A large monolayer suspension was created by injecting monosized melamine formaldehyde microparticles (diameter $9.19 \pm 0.09 \mu \mathrm{m})$. The monolayer was kept for the 2 sets

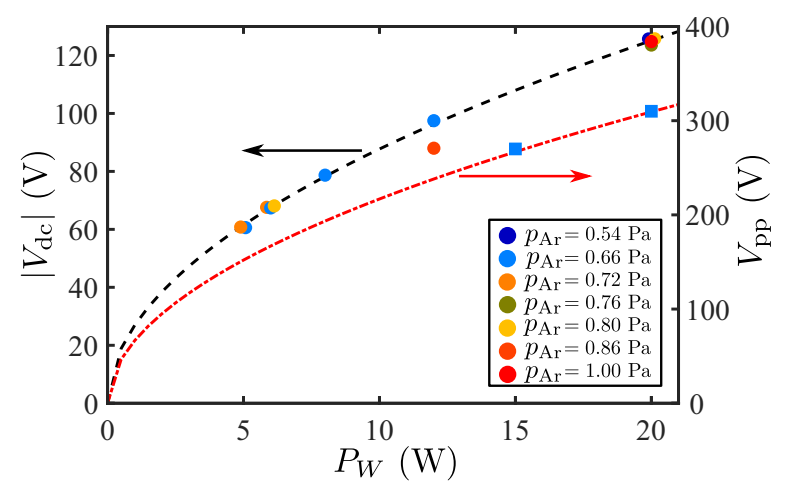

FIG. 3. (०) Self-bias voltage $\left|V_{\mathrm{dc}}\right|$ as a function of rf power, $P_{\mathrm{W}}$, for different working pressures (color coded). ( $\square$ ) Peak-to-peak rf voltage, $V_{\mathrm{pp}}$, as a function of rf power, $P_{\mathrm{W}}$, at $p_{\mathrm{Ar}}=0.66 \mathrm{~Pa}$. The dashed black line is the fit $\left|V_{\mathrm{dc}}\right|=27 \times P_{\mathrm{W}}^{0.51}$. The dotted red line is $V_{\text {pp }}$ calculated from Eq. (1).
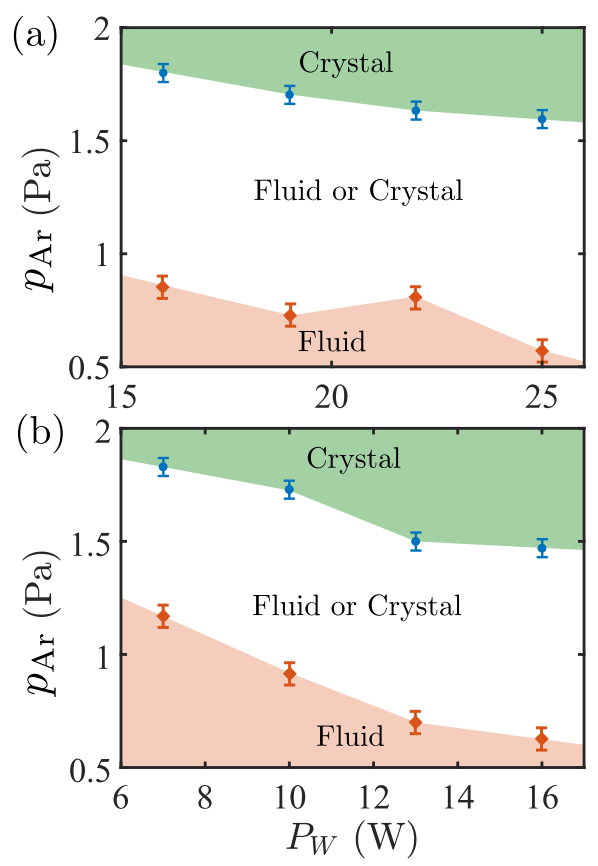

FIG. 4. Crystallization pressure, $p_{\text {cryst }}$ (blue dots), and MCI threshold pressure, $p_{\mathrm{MCI}}$ (red diamonds), for different rf powers, $P_{\mathrm{W}}$ : (a) experiment I, (b) experiment II. Above the crystallization pressure the monolayer is always in the crystalline state (green areas). Below the MCI threshold pressure, the monolayer is always in the fluid state. In between, the monolayer remains in the crystalline phase when decreasing the pressure from above the crystallization threshold and remains fluid when increasing the pressure from below the MCI threshold.

of experiments (referred to as experiment I and experiment II) separated by $\sim 1$ hour. According to Ref. [51], the MF microparticles are etched at a rate of $\sim 1.25 \mathrm{~nm} / \mathrm{min}$ in an argon discharge meaning that the microparticle diameter for the second set of experiments was $\sim 9 \mu \mathrm{m}$.

The crystallization and melting pressures of the monolayer were explored for different rf powers. At a given rf power, starting from a fluid monolayer, the argon pressure was gradually increased until the monolayer was fully crystallized (pressure step of $\sim 0.6$ mTorr) and the crystallization pressure was recorded. Note that during the pressure increase period, the monolayer remained in the fluid phase [52]. The dynamics of the microparticles at the crystallization threshold was not investigated in details and the crystallization event was identified with the naked eye (see Appendix A). Then the pressure was very slowly decreased until MCI was triggered in the crystal (pressure step of $\sim 0.6$ mTorr when close to the crystallization threshold gradually reduced to $\sim 0.06$ mTorr (at best) when getting closer to MCI threshold) resulting in the rapid melting of the monolayer in a pattern similar to the results reported in Refs. [29-32,53,54]. Note that during the pressure decrease period, the monolayer remained in the crystalline phase until MCI was triggered. The rf power was then decreased and the procedure was repeated.

The results for the two sets of experiments are shown in Fig. 4. As can be seen, in both cases, the higher the power the lower the crystallization and melting pressures. Moreover, 
the gap $\Delta p=p_{\text {cryst }}-p_{\mathrm{MCI}}$, in which the monolayer remains in the crystalline phase when decreasing the pressure from above the crystallization threshold and remains fluid when increasing the pressure from below the MCI melting threshold, increases when increasing the rf power.

During the decreasing pressure phase, the crystalline monolayer was imaged [55] with the high-speed Photron camera in order to track the particle trajectories, measure the crystalline monolayer kinetic temperature $T_{\text {cryst }}$ by fitting the microparticle velocity distribution function by a Maxwellian distribution function, and recover the longitudinal and transverse current fluctuation spectra following the procedure described in Refs. [53,56,57]. To extract the longitudinal and transverse dispersion relations $\omega_{\mathrm{L}, \mathrm{T}}(k)=2 \pi f_{\mathrm{L}, \mathrm{T}}(k)$ where $f_{\mathrm{L}, \mathrm{T}}(k)$ is the frequency of the lattice modes with wave number $k$, the current fluctuation spectra $J_{\mathrm{L}, \mathrm{T}}(k, f)$ were fitted to a double-Lorentzian form $[58,59]$ :

$$
\begin{aligned}
J_{\mathrm{L}, \mathrm{T}}(k, \omega) \propto & \frac{A(k)}{\left(\omega-\omega_{\mathrm{L}, \mathrm{T}}\right)^{2}+A(k)} \\
& +\frac{A(k)}{\left(\omega+\omega_{\mathrm{L}, \mathrm{T}}\right)^{2}+A(k)},
\end{aligned}
$$

with $A(k)$ a constant proportional to the damping rate of the mode. The indices $L$ and $T$ are for longitudinal and transverse, respectively. In Fig. 5(a), the fit of the current spectrum for a normalized wave number $k \Delta=1.3$, where $\Delta$ is the interparticle distance, is shown, and the entire experimental longitudinal dispersion relation is shown in Fig. 5(b) for a wave propagating at an angle of $0^{\circ}$ with respect to the camera field of view. The interparticle distance $\Delta$ was obtained from the position of the first peak of the pair correlation function and error bars are the widths of the peaks at half maximum. Due to high noise level for $k \Delta<0.25$, the frequencies of the mode at these long wavelengths were not measured. Then, longitudinal and transverse dispersion relations were fitted for $k \Delta<1.5$ by the polynomial of the form $\omega_{\mathrm{L}, \mathrm{T}}=C_{\mathrm{L}, \mathrm{T}}|k|+$ $b_{\mathrm{L}, \mathrm{T}}|k|^{3}$, where $C_{\mathrm{L}, \mathrm{T}}$ are the longitudinal and transverse sound speeds and $b_{\mathrm{L}, \mathrm{T}}$ are constants taking into account the nonlinearity of the dispersion relation for $|k \Delta| \gtrsim 1$.

By numerically rotating the frame with respect to the original camera field of view and repeating the procedure, wave propagation could be studied at different angles. $C_{\mathrm{L}, \mathrm{T}}$ were obtained every $5^{\circ}$ [see Fig. 5(c)] and the average values of the sound speeds were calculated for different crystal conditions. Note that the camera field of view contained many crystallites with different orientations. This is not an issue since sound speeds are quasi-independent of the direction of propagation in the crystal $[60,61]$. Assuming screened Coulomb interactions and following the method of Ref. [6], these values were then used to extract the microparticle charge $Z_{d}$, the Debye (screening) length $\lambda_{d}$, and the coupling parameter $\kappa=\Delta / \lambda_{d}$. The results are summarized in Table I. As can be seen, decreasing the pressure at constant rf power resulted in an increase of both sound speeds and a slight increase of the interparticle distance. The microparticle kinetic temperature showed no clear trends and remained quite low while the monolayer was in the crystal phase $\left(T_{\text {cryst }} \lesssim 0.2 \mathrm{eV}\right)$. Assuming pure screened Coulomb interactions, it corresponded to an increase of the microparticle charges. Accordingly, the max-
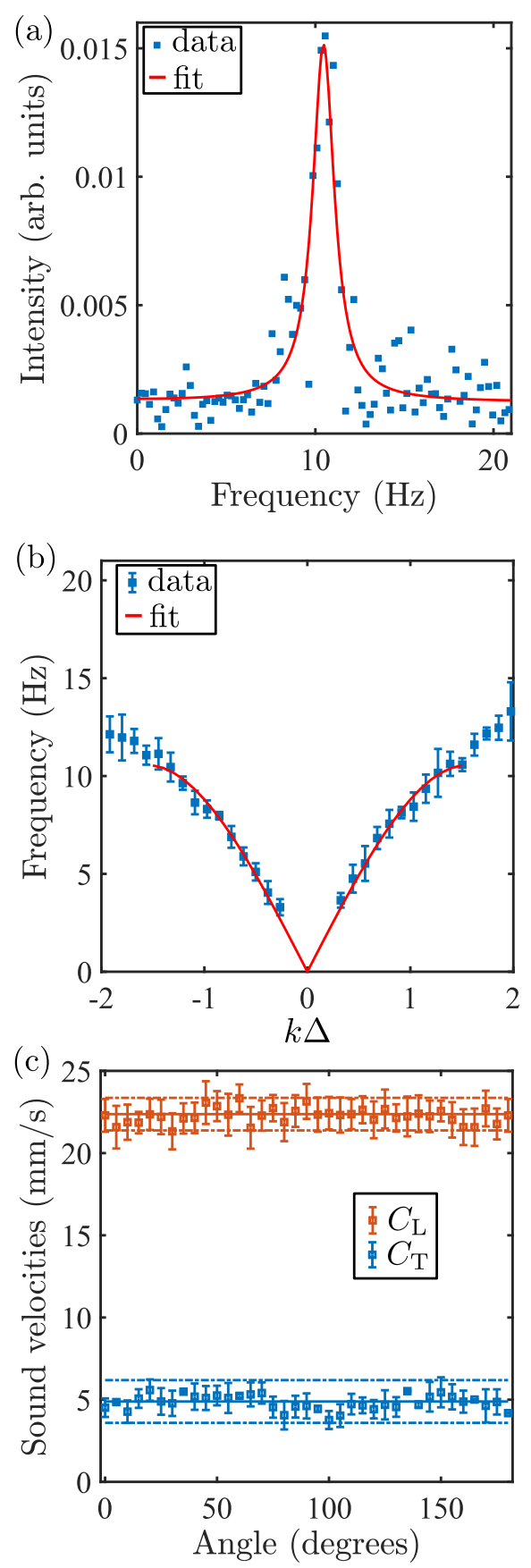

FIG. 5. (a) Fit of the longitudinal current fluctuation spectrum for $k \Delta=1.3$. Only the "positive" frequency side of the Fourier transform is shown. (b) Fit of the reconstructed longitudinal dispersion relation for wave propagating at an angle of $0^{\circ}$ with respect to the camera field of view. (c) Longitudinal sound speed, $C_{\mathrm{L}}$, and transverse sound speed, $C_{\mathrm{T}}$, measured for different wave propagation angles with respect to the camera field of view. All plots correspond to measurements at $P_{\mathrm{W}}=16 \mathrm{~W}$ and $p=1.80 \mathrm{~Pa}$.

imum frequency of the longitudinal dispersion relation (not shown here) increased when decreasing pressure making the out-of-plane mode and the in-plane mode closer to each other as the MCI pressure threshold, $p_{\mathrm{MCI}}$, was approached. No trend in the evolution of the coupling parameter $\kappa=\Delta / \lambda_{\mathrm{D}} \simeq$ $1.0-1.8$ could be extracted due to the large error on the sound 
TABLE I. Two-dimensional crystal parameters for different discharge conditions between the crystallization pressure, $p_{\text {cryst }}$, and the MCI threshold pressure, $p_{\mathrm{MCI}}$. The microparticle charge $Z_{d}$ and the Debye (screening) length $\lambda_{d}$ were calculated assuming screened Coulomb interactions [6]. The crystalline monolayer kinetic temperature $T_{\text {cryst }}$ was measured by fitting the microparticle velocity distribution function by a Maxwellian distribution function.

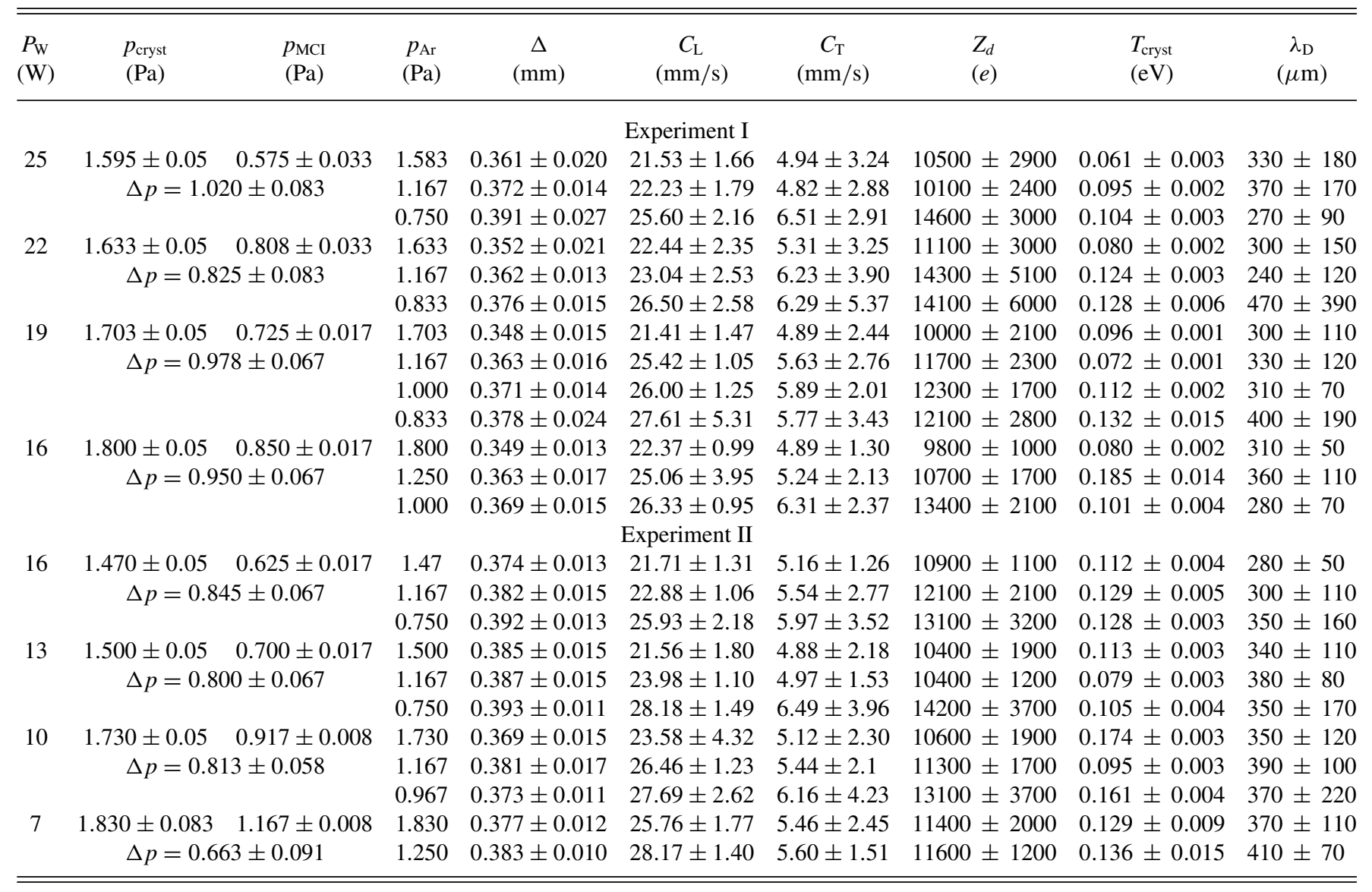

speeds, especially the transverse sound speed. Note that the coupling parameter $\Gamma=\left(Z_{d}^{2} e\right) /\left(T_{\text {cryst }} 4 \pi \epsilon_{0} \Delta\right) \sim 2000$, where $e$ is the elementary charge and $\epsilon_{0}$ the vacuum permittivity, remains far from the coupling parameters at the melting point, $\Gamma_{m} \sim 200$ at $\kappa \sim 1.2$ [62].

\section{SHEATH PROFILE ABOVE THE POWERED ELECTRODE OF A CAPACITIVELY COUPLED RADIO-FREQUENCY DISCHARGE}

Any modification of the discharge parameters $\left(p_{\text {Ar }}\right.$ and/or $\left.P_{\mathrm{W}}\right)$ has an impact on the plasma and therefore on the sheath properties. It is then evident that since the microparticle monolayer levitates in the rf sheath above the powered electrode, any changes of the discharge parameters also affect the monolayer properties. In this section, a simple model allowing calculation of the sheath profile above the powered electrode in an asymmetric cc-rf discharge as a function of the discharge parameters is described. The model is then validated against available experimental data and then used to obtain the dependence of sheath parameters (sheath length, ion and electron densities, electric field) as a function of the discharge parameters $\left(p_{\mathrm{Ar}}\right.$ and $\left.P_{\mathrm{W}}\right)$.

\section{A. Description of the model}

In a cc-rf discharge, the rf frequency is generally much higher than the ion plasma frequency and much lower than the electron plasma frequency, i.e., $\omega_{\text {pi }} \ll \omega_{\text {rf }} \ll \omega_{\text {pe }}$, where $\omega_{\mathrm{pi}}, \omega_{\mathrm{pe}}, \omega_{\mathrm{rf}}$ are the ion, electron, and $\mathrm{rf}$ angular frequency, respectively. In that respect, the ions respond to the timeaveraged sheath electric field. Moreover, in most of 2D complex plasma crystal experiments, the working pressure is around $1 \mathrm{~Pa}$. In this situation, ion collisions cannot be neglected [35]. The main assumptions of the following model are based on earlier work by Lieberman [35]:

(i) collisional ion motion with a constant ion mean free path $\lambda_{i}$ in the sheath,

(ii) cold ions (ion temperature $T_{i} \simeq 0$ ),

(iii) inertia-less electrons that respond instantaneously to the electric field,

(iv) no secondary electrons emitted from the electrode,

(v) no ionization in the sheath.

In most experiments, asymmetric cc-rf discharges with grounded area much larger than the powered electrode area are used. Moreover, since the matching circuit very often contains a blocking capacitor, no direct current can flow in the external circuit. Since the area of each electrode is different, the amount of collected ions and electrons during each 
rf cycle is also different and results in the appearance of a self-bias voltage on the powered electrode [37,48]. Ideally, it would be necessary to consider both sheaths in front of both electrodes simultaneously [37]. However, in order to simplify the problem, the following assumptions are made:

(i) The voltage on the rf electrode is of the form

$$
V_{\mathrm{rf}}(t)=-V_{0} \cos \left(\omega_{\mathrm{rf}} t\right)-\left|V_{\mathrm{dc}}\right| .
$$

The input values of $V_{0}$ and $V_{\mathrm{dc}}$ were discussed in Sec. III.

(ii) At all time instants, the plasma must remain quasineutral and therefore the current going through the powered electrode sheath and the anode sheath must be equal in intensity and of opposite signs. Following the method described by Song et al. [49], the time dependence of the plasma potential with respect to the grounded wall is approximated by

$$
\begin{aligned}
V_{p}(t)= & T_{e} \ln \left\{\left[1+\frac{A_{\mathrm{rf}}}{A_{g}} \exp \left(\frac{V_{\mathrm{rf}}(t)}{T_{e}}\right)\right]\right. \\
& \left.\times\left(1+\frac{A_{\mathrm{rf}}}{A_{g}}\right)^{-1}\right\}+\frac{T_{e}}{2} \ln \left(\frac{M}{2.3 m_{e}}\right),
\end{aligned}
$$

where $T_{e}$ is the electron temperature in $\mathrm{eV}, m_{e}$ is the electron mass, and $M$ is the ion mass.

In the following, the plasma potential is taken as the reference potential $\left(V_{p}=0\right)$. At any given instant of time, the potential of the rf electrode $V_{c}$ with respect to the plasma is

$$
V_{c}(t)=V_{\mathrm{rf}}(t)-V_{p}(t)
$$

The time-dependant potential profile $V(z, t)$ in the sheath follows the Poisson equation:

$$
\nabla^{2} V(z, t)=-\frac{e}{\epsilon_{0}}\left[n_{\mathrm{i}}(z)-n_{e}(z, t)\right],
$$

where $n_{\mathrm{i}(\mathrm{e})}(z)$ is the ion (electron) density. The boundary conditions are $V(0, t)=0$ and $V\left(\ell_{s}, t\right)=V_{c}(t)$, where $\ell_{s}$ is the length of the ion sheath.

Since $\omega_{\mathrm{pi}} \ll \omega_{\mathrm{rf}}$, ions react only to the time-averaged electric field $\bar{E}=-d \bar{V} / d z$ where $\bar{V}$ is the time-averaged electric potential across the sheath of the powered electrode. Therefore, in the sheath, the ion particle conservation and momentum conservation equations are $[36,63]$

$$
\begin{gathered}
n_{0} v_{s}=n_{i} v_{i}, \\
M v_{i} \frac{d v_{i}}{d z}+e \frac{d \bar{V}}{d z}+\frac{\pi M v_{i}^{2}}{2 \lambda_{i}}=0,
\end{gathered}
$$

where $n_{0}$ is the plasma density at the plasma sheath boundary, $v_{i}$ is the ion velocity at a given position $z$ in the sheath, and $v_{s}$ is the velocity of the ions at the ion sheath boundary $(z=0)$. When taking into account collisions, $v_{s}$ is the modified Bohm velocity $[36,63]$ :

$$
v_{s}=\left(\frac{e T_{e}}{M}\right)^{1 / 2}\left(1+\frac{\pi \lambda_{\mathrm{D}_{e}}}{2 \lambda_{i}}\right)^{-1 / 2}
$$

where $\lambda_{\mathrm{D}_{\mathrm{e}}}=\left(\epsilon_{0} T_{e} / n_{0} e\right)^{1 / 2}$ is the electron Debye length.

In order to calculate the sheath profile, one needs to make a few approximations. First of all, in order to solve Eq. (6), one needs to know the exact position of the time-varying sheath boundary. Indeed, depending on the voltage across the sheath,

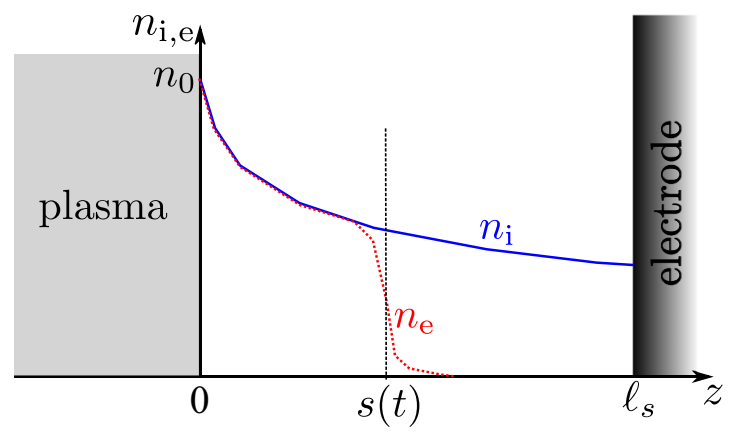

FIG. 6. Schematic of a capacitive rf sheath at a time $0<t<$ $\pi / \omega_{\mathrm{rf}}$. The dotted vertical line shows the position of the electron sheath boundary $s(t)$. For $z<s(t), n_{e} \sim n_{i}$ and for $z>s(t), n_{e} \sim 0$.

the electron sheath boundary $s(t)$ will change and oscillate between the position of the ion sheath boundary to a minimum distance to the electrode (see Fig. 6). Generally, in cc-rf discharges, the amplitude of the rf voltage is much higher than the electron temperature, i.e., $V_{0} \gg T_{e}$, and the electron Debye length is much smaller than the sheath width $\ell_{s}$. It is thus clear that on the plasma side $[z<s(t)]$, the quasineutrality holds $\left(n_{e} \sim n_{i}\right)$, while on the sheath side $[z>s(t)]$, the electron density falls very rapidly $\left(n_{e} \sim 0\right)$. Many studies dealing with the structure of cc-rf sheath therefore use this approximation known as the step model which has been extensively used in different models of the cc-rf sheath [34-40,42]. In many of these models, the solution of the rf sheath relies on calculating the rf current through the sheaths (powered and grounded) and assuming a zero net current [34-37,40]. It allows one to calculate the position of the electron sheath at all times and from there the other quantities such as the average potential drop and average electric field in the sheath can be derived. Calculations of these kind have been performed for both symmetric [34-36] and asymmetric discharges [37,40]. It was nevertheless shown that, in the case of symmetric cc-rf discharges, these models were able to accurately describe the current-voltage characteristics but showed discrepancies in the potential and electric field profiles with respect to the exact solution [38]. More recent models allow for very accurate descriptions of the cc-rf sheath but require input such as the maximum sheath extension, the ion mean free path, and the electron Debye length [42]. The ion mean free path and the electron Debye length can be easily obtained from discharge pressure, interferometry measurements, and literature data. However, the maximum sheath extension is not a parameter which is very often measured. Moreover, the current and voltage wave forms on the powered electrode are usually not monitored. However, in our experiments, the self-bias voltage and the electron density were measured as a function of the forward rf power and argon pressure over a wide range of parameters which can be used to recover the rf voltage amplitude $V_{0}$ using Eq. (1). The electron temperature can be estimated using the uniform density discharge model [47]:

$$
\frac{K_{i z}\left(T_{e}\right)}{u_{\mathrm{B}}\left(T_{e}\right)}=\frac{1}{n_{g} d_{\mathrm{eff}}},
$$

where $K_{i z}\left(T_{e}\right)=2.34 \times 10^{-14} \times T_{e}^{0.59} \times \exp \left(-17.44 / T_{e}\right)$ is the ionization rate [47], $u_{\mathrm{B}}\left(T_{e}\right)=\sqrt{e T_{e} / M}$ is the Bohm 
velocity, $n_{g}$ is the neutral gas number density, and $d_{\mathrm{eff}}=0.5 \times$ $R l /\left(R h_{l}+l h_{r}\right)$ is the effective plasma size for particle loss with $l$ the height of the discharge chamber, $R$ its radius, $h_{r}=$ $0.80\left(4+R / \lambda_{\text {in }}\right)^{-1 / 2}$ and $h_{l}=0.86\left[3+l /\left(2 \lambda_{\text {in }}\right)\right]^{-1 / 2} \quad[47]$, and $\lambda_{\text {in }}$ the ion mean free path in the plasma bulk.

The sheath profile can then be calculated using the following approximation for the time-dependent Poisson equation:

$$
\nabla^{2} V(z, t) \simeq-\frac{e n_{i}(z)}{\epsilon_{0}}\left(1-\exp \frac{V(z, t)}{T_{e}}\right) .
$$

As can be seen, this approximation lies between the ion matrix sheath approximation $\left[n_{i}(z)=n_{0}\right]$ and the step model. It however allows us to solve the Poisson equation at anytime knowing the voltage drop in the sheath. The mean electric field and ion density profiles are then obtained in a recursive approach. Starting from the collision-less dc sheath profile with a potential drop equal to the maximum of $V_{c}(t)$, a maximum sheath length and an initial ion density profile are calculated [47,64]:

$$
\begin{gathered}
\ell_{s}=\frac{\sqrt{2}}{3} \lambda_{\mathrm{D}_{\mathrm{e}}}\left(\frac{2 \max \left[\left|V_{c}(t)\right|\right]}{T_{e}}\right)^{3 / 4}, \\
\bar{V}(z)=-\max \left[\left|V_{c}(t)\right|\right]\left(\frac{z}{\ell_{s}}\right)^{4 / 3}, \\
n_{i}(z)=n_{0}\left(1-\frac{2 \bar{V}(z)}{T_{e}}\right)^{-1 / 2} .
\end{gathered}
$$

Then the time-dependant potential profile over one rf period is calculated by solving Eq. (11) with MATLAB and using the following boundary conditions:

$$
\begin{gathered}
V(0, t)=0, \\
V\left(\ell_{s}, t\right)=V_{c}(t),
\end{gathered}
$$

and a new time-averaged potential and electric field are calculated. If the time-averaged electric field on the plasma side $(z=0)$ is lower than $E_{\min }=(\pi / 2)\left(T_{e} / \lambda_{i}\right)$, a value necessary to ensure monotonically decreasing ion density and increasing ion velocity in the sheath region, then the sheath boundary on the plasma side is moved toward the wall until the average electric field reaches $E_{\min }$ and a new sheath length $\ell_{s}$ is obtained. Using Eqs. (7) and (8) with the boundary conditions

$$
\begin{aligned}
& n_{i}(0)=n_{0}, \\
& v_{i}(0)=v_{s},
\end{aligned}
$$

a new ion density profile is obtained and the time-dependant potential profile is calculated again. The procedure is repeated until it converges, which typically occurs in 10 to 15 iterations.

\section{B. Comparison to experimental data, validation of the model}

Schulze et al. have measured experimentally the timedependant electric field of a cc-rf capacitive discharge in krypton at different pressures [65]. Czarnetzki used this set of measurements to validate with success his advanced model of the cc-rf sheath [42].

Two sets of discharge parameters were used to validate our model:
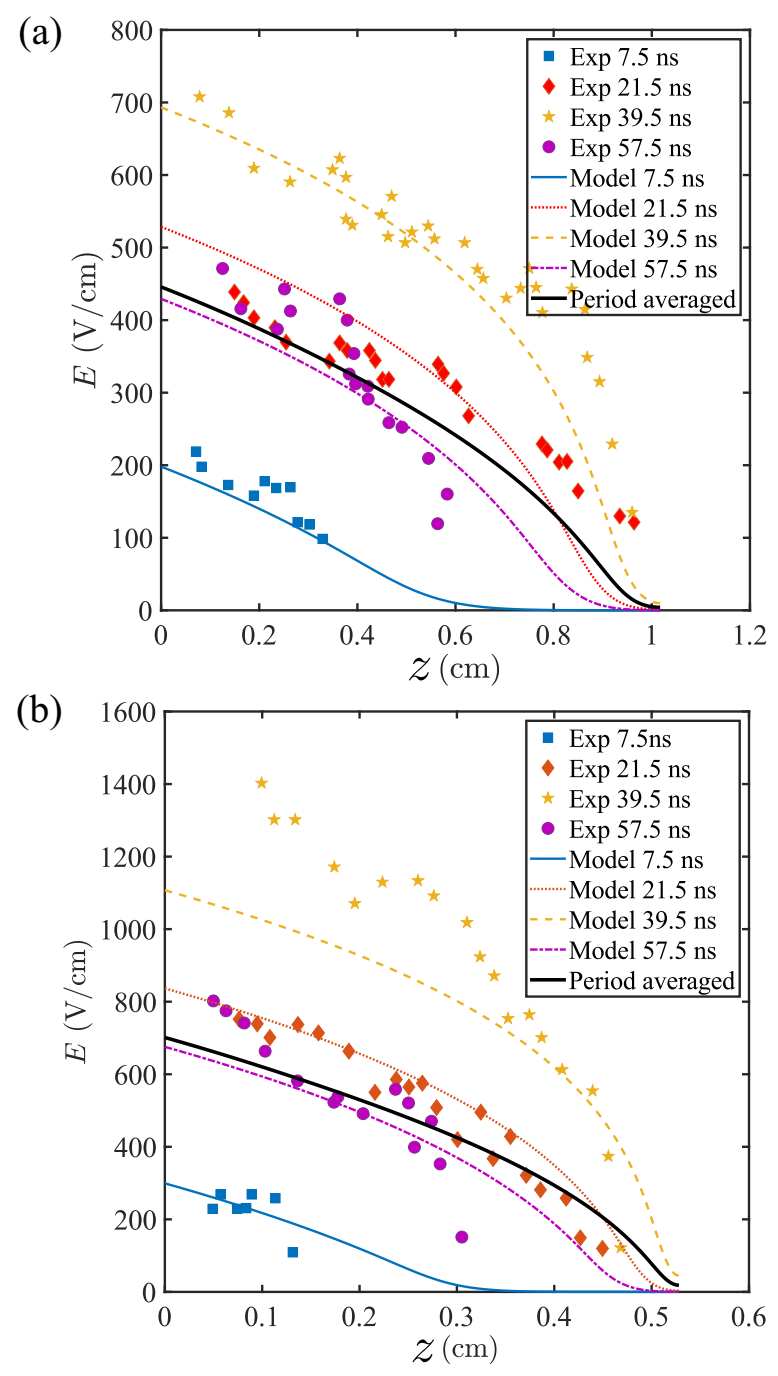

FIG. 7. Experimental time-resolved electric field in krypton cc-rf discharge compared to the calculated electric field using the model described in this article. The experimental data are extracted from Ref. [65]. (a) $p_{\mathrm{Kr}}=1 \mathrm{~Pa}$ and $P_{\mathrm{rf}}=8 \mathrm{~W}$, (b) $p_{\mathrm{Kr}}=10 \mathrm{~Pa}$ and $P_{\mathrm{rf}}=$ $8 \mathrm{~W}$. The time instants are given in the inset and in both plots the plain black line corresponds to the time-averaged electric field.

(i) $p_{\mathrm{Kr}}=1 \mathrm{~Pa}$ and $P_{\mathrm{rf}}=8 \mathrm{~W}$, corresponding to $V_{0} \simeq$ $250 \mathrm{~V}$ and $V_{\mathrm{dc}} \simeq-250 \mathrm{~V}$ (see Fig. 12 of Ref. [65]). As in Ref. [42], the electron temperature is fixed to $T_{e}=2.6 \mathrm{eV}$ and the plasma density at the sheath edge to $n_{0}=2.0 \times 10^{15} \mathrm{~m}^{-3}$. The grounded surface is assumed to be 100 times larger than the powered surface. The charge exchange cross section is $\sigma_{C X}=40 \times 10^{-20} \mathrm{~m}^{2}[66]$.

(ii) $p_{\mathrm{Kr}}=10 \mathrm{~Pa}$ and $P_{\mathrm{rf}}=8 \mathrm{~W}$, corresponding to $V_{0} \simeq$ $225 \mathrm{~V}$ and $V_{\mathrm{dc}} \simeq-225 \mathrm{~V}$ (see Fig. 5 of Ref. [65]). As in Ref. [42], the electron temperature is fixed to $T_{e}=1.1 \mathrm{eV}$ and the plasma density at the sheath edge to $n_{0}=7.3 \times 10^{15} \mathrm{~m}^{-3}$. The grounded surface is assumed to be 100 times larger than the powered surface. The charge exchange cross section is $\sigma_{C X}=40 \times 10^{-20} \mathrm{~m}^{2}[66]$.

In Fig. 7, the model results are compared to the experimental data of Schulze et al. [65]. As can be seen, the agreement is not perfect and the model tends to slightly underestimate the electric field, especially at large pressure. However, one 


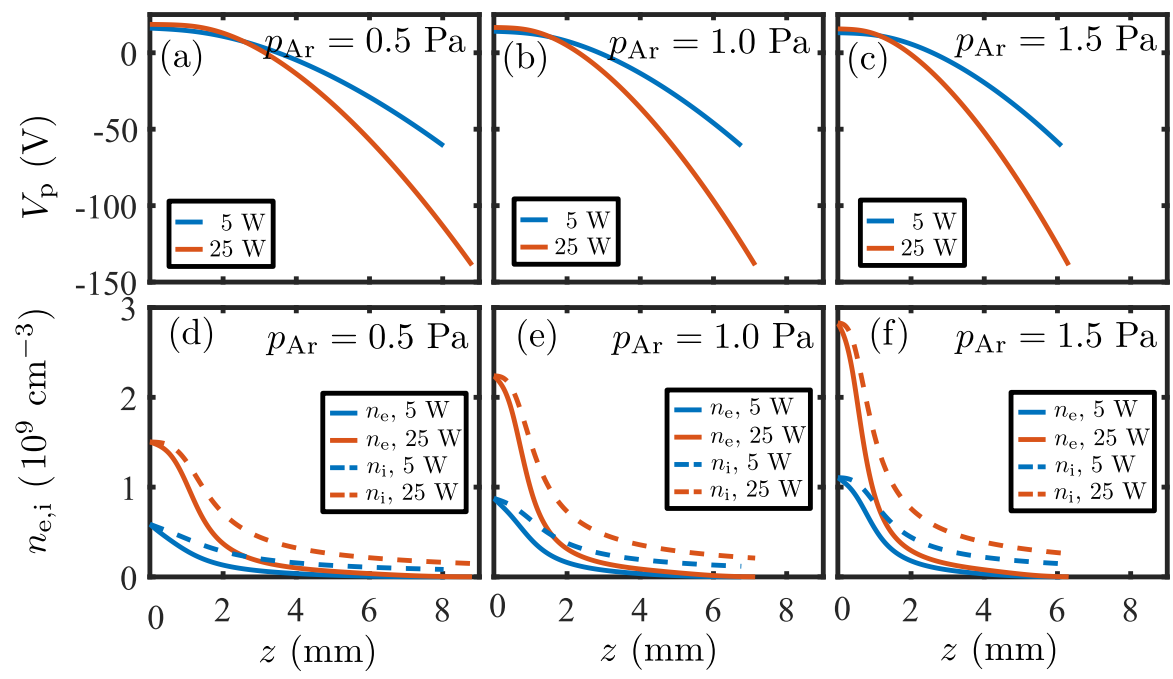

FIG. 8. (a)-(c) Potential and (d)-(f) density profiles for different pressures and powers. Note that potentials are plotted with respect to ground [i.e., $\left.V_{\mathrm{p}}(0) \neq 0\right]$.

should note that the length of the sheath is recovered within a few percent and that the electric field values are in respectable agreement with the measured ones.

We can therefore conclude that our simple model is able to catch the main features of the collisional cc-rf sheath and can reasonably estimate the main sheath parameters as a function of position for an adequate range of discharge parameters.

\section{Calculated sheath profile}

The model was then used for conditions relevant to our experimental conditions. The argon pressure was varied between $0.5 \mathrm{~Pa}$ and $2 \mathrm{~Pa}$ by steps of $0.05 \mathrm{~Pa}$ and the rf power was varied between $5 \mathrm{~W}$ and $25 \mathrm{~W}$ by steps of $1 \mathrm{~W}$. In Fig. 8 the sheath potential [Figs. 8(a)-8(c)] and the electron and ion densities [Figs. 8(d)-8(f)] are shown as a function of position for different pressures and rf powers.

As can be seen in the top row of Fig. 8, increasing pressure at constant rf power results in shorter sheath length for a similar potential drop (the sheath length is $\sim 8-9 \mathrm{~mm}$ at $p=0.5 \mathrm{~Pa}$ and drops down to $\sim 6-6.5 \mathrm{~mm}$ at $p=1.5 \mathrm{~Pa}$ ). Therefore, the vertical electric field is weaker at lower pressures. One can therefore expect a weaker vertical confinement for microparticles levitating in the rf sheath at low pressure for a given rf power. Increasing the rf power at constant pressure results in a slight increase of the sheath length but larger plasma potential drops due to increasing self-bias voltage (the potential drop is $\sim 50 \mathrm{~V}$ at $P_{\mathrm{W}}=5 \mathrm{~W}$ and $\sim 140 \mathrm{~V}$ at $\left.P_{\mathrm{W}}=25 \mathrm{~W}\right)$. Therefore, a stronger vertical electric field and a stiffer vertical confinement for microparticles levitating in the rf sheath at high rf power are expected.
Both $P_{\mathrm{W}}$ and $p_{\text {Ar }}$ have an impact on plasma density. We know that at a given pressure, the plasma density is increasing almost linearly with rf power (see Fig. 2). Since the sheath length is weakly dependant on rf power (see previous paragraph), the gradients of ion and electron densities in the sheath increase with rf power as can be seen in the bottom row of Fig. 8. At high pressure, the plasma density is higher (due to collisions) and the sheath length is short, resulting in steep density gradients.

Using the calculated sheath profiles, the dependence of dust particle charge number, $Z_{d}$, levitation height, $z_{\text {lev }}$, and vertical resonance frequency, $f_{\mathrm{v}}$, on discharge parameters is explored in the next section.

\section{MICROPARTICLE PROPERTIES AND MODE-COUPLING INSTABILITY}

In this section, the influence of discharge parameters on $Z_{d}$, $z_{\text {lev }}$, and $f_{\mathrm{v}}$ is investigated. Then, the threshold of MCI in 2D complex plasma crystal as a function of discharge parameters is studied.

\section{A. Dust particle charge, levitation height, and vertical resonance frequency}

With the calculated sheath profile as a function of $\mathrm{rf}$ power and pressure, the equilibrium dust particle charge and levitation height can be calculated. At any point in the sheath, the ion and electron densities are known allowing the calculation of ion and electron currents, $I_{i}$ and $I_{e}$, respectively, onto the microparticle surface in a collisional plasma $[67,68]$ :

$$
\begin{gathered}
I_{e}=(-e) \sqrt{8 \pi} r_{d}^{2} n_{e} v_{T_{e}} \exp (-\tilde{\varphi}), \\
I_{i}=(e) \sqrt{8 \pi} r_{d}^{2} n_{e} v_{T_{i}}(1+\tilde{\varphi})\left[1+\frac{\tilde{\varphi}\left(\frac{T_{e}}{T_{i}} \frac{r_{d}}{\lambda_{i}}\right)}{\left.0.07+2\left(\frac{r_{d}}{\lambda}\right)+2.5\left(\frac{r_{d}}{\lambda_{i}}\right)+\left[0.27\left(\frac{r_{d}}{\lambda}\right)^{1.5}+0.8\left(\frac{r_{d}^{2}}{\lambda_{i} \lambda}\right)\right] \frac{T_{e}}{T_{i}} \tilde{\varphi}+\frac{0.4\left(\frac{r_{d}}{\lambda_{i}}\right)^{2}\left(\frac{T_{e}}{T_{i}}\right) \tilde{\varphi}}{1-0.4\left(\frac{d}{\lambda_{i}}\right)}\right]}\right],
\end{gathered}
$$


(a)

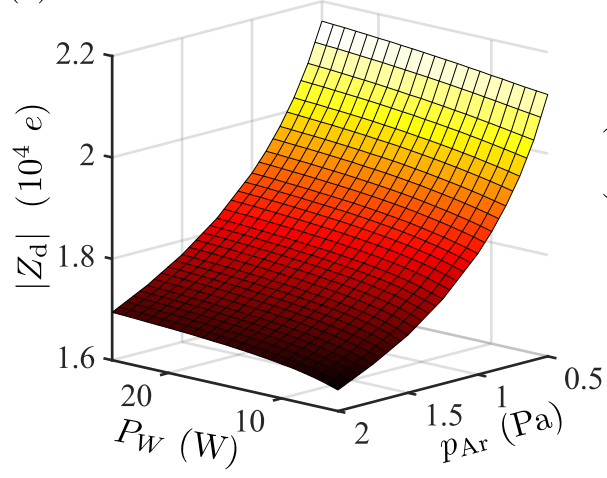

(b)

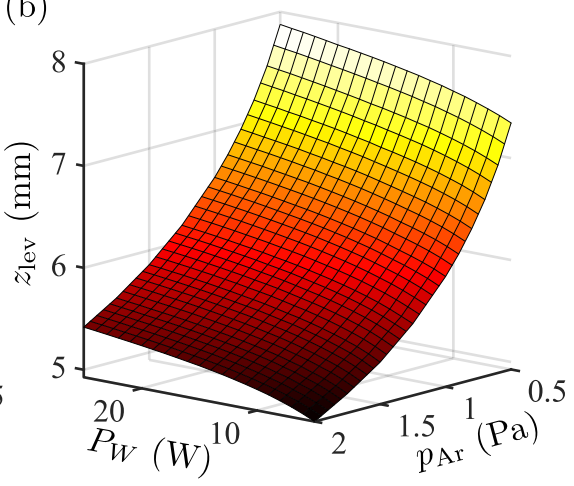

(c)

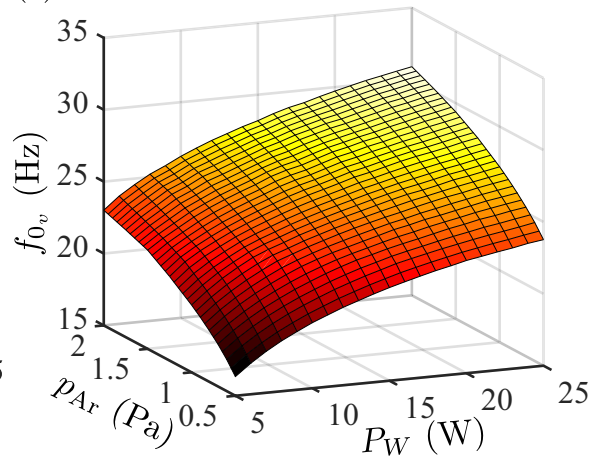

FIG. 9. (a) Equilibrium particle charge. (b) Equilibrium levitation height. (c) Vertical resonance frequency. The microparticle has a diameter $d=9.16 \mu \mathrm{m}$ and its mass density is $\rho_{m}=1510 \mathrm{~kg} \mathrm{~m}^{-3}$ (MF density).

where $r_{d}$ is the radius of the microparticle, $\tilde{\varphi}$ is the normalized microparticle floating potential, and $\lambda=$ $\lambda_{\mathrm{D}_{\mathrm{i}}} / \sqrt{1+\left(\lambda_{\mathrm{D}_{\mathrm{i}}} / \lambda_{\mathrm{D}_{\mathrm{e}}}\right)^{2}}$ is the linearized Debye length at the considered position in the sheath with $\lambda_{\mathrm{D}_{\mathrm{i}, \mathrm{e}}}=\left(\epsilon_{0} T_{\mathrm{i}, \mathrm{e}} / n_{\mathrm{i}, \mathrm{e}} e\right)^{1 / 2}$. At equilibrium $I_{i}+I_{e}=0$ and the normalized floating potential $\tilde{\varphi}$ can be obtained for any position in the sheath (ions are assumed to be in thermal equilibrium with the background gas, $T_{i}=0.03 \mathrm{eV}$ ). The microparticle charge number $Z_{d}=$ $Q_{d} / e$, where $Q_{d}$ is the microparticle charge, is then obtained:

$$
Z_{d}=-4 \pi \epsilon_{0} r_{d} T_{e} \tilde{\varphi} / e .
$$

Since $r_{d}$ and therefore the mass $m_{d}$ of the microparticle are known and since the sheath electric field profile has been calculated, the equilibrium levitation height $z_{\text {lev }}$ and the vertical resonance (confinement) frequency $f_{\mathrm{v}}=\omega_{v} /(2 \pi)$ can be obtained using the following equations [69-72]:

$$
\begin{gathered}
Q_{d} E\left(z_{\mathrm{lev}}\right)=-m_{d} g, \\
\omega_{v}=\sqrt{\frac{\left|Q_{d}\right|}{m_{d}} \frac{\partial E(z)}{\partial z}} \underset{\mid z=z_{\mathrm{lev}}}{ } .
\end{gathered}
$$

In Fig. 9, the results of the calculation of levitation height, particle charge at equilibrium position, and vertical resonance frequency as a function of rf power and background argon pressure are shown. The obtained equilibrium particle charges are between $-2.2 \times 10^{4} e$ and $-1.6 \times 10^{4} e$ [see Fig. 9(a)], which is of the order of the experimental ones (see Table I).

One can see that at a given argon background pressure, $Z_{d}$ and $z_{\text {lev }}$ are only slightly increasing with rf power. Moreover, the microparticles levitate quite high in the sheath near the sheath edge (for example at $1 \mathrm{~Pa}$, the sheath length is around $\sim 7 \mathrm{~mm}$ (see Fig. 8) and the levitation height is around $\sim 6.5 \mathrm{~mm}$ [see Fig. 9(b)]). This is due to the fact that even though the ion and electron densities are increasing with rf power, the electron temperature is mostly set by the background gas pressure. Since $T_{e}$ has a greater influence than $n_{e}$ on the magnitude of the electron current onto a microparticle and therefore the microparticle charge, the microparticle equilibrium charge depends only weekly on rf power. Since at equilibrium levitation height, the gravity force is compensated by the electric force and, as seen previously, the sheath length is only weakly dependant on rf power, the particle levitation height will only slightly change.

The calculated vertical resonance frequency varies in the range $15-30 \mathrm{~Hz}$ depending on discharge parameters and is very close to the vertical resonance frequency reported in similar setups $[9,10,28,73-75]$. The vertical resonance frequency is increasing significantly with both rf power and argon background pressure [see Fig. 9(c)]. In both cases, this is due to steeper electric field when increasing rf power (resulting in larger self-bias) and when increasing the background argon pressure (resulting in shorter sheath). Therefore, decreasing pressure or $\mathrm{rf}$ power results in weaker vertical confinement and favors MCI for 2D complex plasma crystals. On the contrary, high pressure and high rf power favor crystallization of the complex plasma monolayer by providing stiffer vertical confinement. More details are given in the next subsection.

\section{B. Mode-coupling instability}

It is well known that MCI can occur in 2D complex plasma crystals $[9,24,25]$ and in fluid complex plasma monolayers $[26,29,32]$. In Sec. IV, it was demonstrated experimentally that decreasing pressure and/or rf power leads to MCI in 2D complex plasma crystals and that the MCI threshold and crystallization threshold have defined trends.

One of the simplest ways to simulate MCI is to use the point-charge wake model [22,24,25,76-78]. In this model, the ion wake is approximated by a positive point charge $q_{w}$ downstream of each microparticle at a distance $\delta_{w}$ below the microparticle. Then each microparticle interacts with the other microparticles and their respective ion wakes through screened-Coulomb (Yukawa) interactions. The interparticle distance in the $2 \mathrm{D}$ crystal is set at a fixed value $\Delta$. In the calculation, the screening length is taken as the electron Debye length, $\lambda_{D_{e}}$ at the equilibrium leviation height. To compute the different lattice modes in the 2D complex plasma crystals, one can calculate the dynamical matrix which has the form $[24,25,78]$

$$
\mathbf{D}=\left(\begin{array}{ccc}
\alpha_{h}-\beta & 2 \gamma & \iota \sigma_{x} \\
2 \gamma & \alpha_{h}+\beta & l \sigma_{y} \\
\iota \sigma_{x} & l \sigma_{y} & \omega_{v}^{2}-2 \alpha_{v}
\end{array}\right),
$$




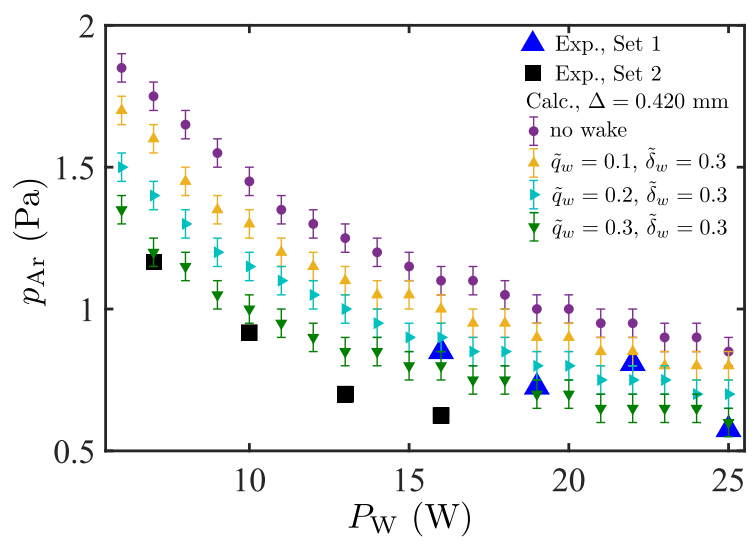

FIG. 10. MCI pressure threshold as a function of rf power for an interparticle distance $\Delta=420 \mu \mathrm{m}$ and different ion wake charges in the framework of the point-charge wake model [24]. The experimental MCI pressure thresholds are shown for comparison.

where $\alpha_{h, v}, \beta$, and $\gamma$ are the dispersion elements, and $\sigma_{x, y}$ are the coupling terms between the in-plane and out-of-plane modes due to the particle-wake interaction. Detailed expressions of these different terms as functions of $Q_{d}, \lambda_{\mathrm{D}_{\mathrm{e}}}, q_{w}$, $\delta_{w}, \Delta$, and $\mathbf{k}$, where $\mathbf{k}$ is the wave vector of the considered lattice mode, can be found in Refs. [24,25,78]. The dispersion relation of the microparticle lattice modes are obtained for any $\mathbf{k}$ by solving $\operatorname{det}[\mathbf{D}-\omega(\omega+\imath v) \mathbf{I}]=0$, where $\mathbf{I}$ is the unit matrix, so that $\omega(\omega+\imath v)$ are the eigenvalues of the dynamical matrix, where $v$ is the damping rate due to neutral gas friction.

It is known that the mode-coupling instability in a $2 \mathrm{D}$ complex plasma crystal is triggered when the out-of-plane mode and the in-plane longitudinal mode cross [24,25] except in very specific experimental conditions where the ion wake charges are really strong allowing coupling of the modes without direct crossing [79]. First, the influence of the interparticle distance $\Delta$ on mode crossing is investigated in the case where ion wakes are ignored (see Appendix B for details). It is found that the out-of-plane and in-plane longitudinal mode crossing minimum pressure decreases when increasing the $\mathrm{rf}$ power due to the competing effects of the pressure decrease and the rf power increase. Moreover, at low pressure the microparticle charge is larger due to a higher electron temperature, which for a fixed $\Delta$ increases the maximum frequency of the longitudinal in-plane mode and makes mode crossing easier. Similarly, the pressure threshold is systematically lower for larger $\Delta$ to the weaker interparticle interaction which reduces the maximum frequency of the longitudinal in-plane mode and the minimum frequency of the out-of-plane mode. Experimental points follow roughly the same trend showing that the sheath profile (which depends on $p_{\mathrm{Ar}}$ and $P_{\mathrm{W}}$ ) plays a major role in the crossing of the modes and the triggering of MCI in 2D complex plasma crystals.

In a second step, the interparticle distance was fixed to $\Delta=$ $420 \mu \mathrm{m}$ and point-charge wakes were considered. Only the value of the wake charge was varied $\left(0.1 \leqslant \tilde{q}_{w}=q_{w} / Q_{d} \leqslant\right.$ $0.3)$, while the distance to the microparticle is fixed $\left(\tilde{\delta}_{w}=\right.$ $\left.\delta_{w} / \lambda=0.3\right)$. Then, the pressure threshold for the crossing of the modes (and the triggering of MCI) were calculated again. The results are presented in Fig. 10. As can be seen, the main trends are still there: when increasing the rf power, the threshold pressure decreases. However, the ion wakes have a significant effect. Increasing the ion wake charge significantly decreases the pressure threshold at a given rf power. The wake charges are unknown in the experiment and their overall effect on the measured pressure threshold are difficult to estimate. However, one can see in Fig. 10 (and Fig. 12 in Appendix B) that the experimental threshold values are not exactly aligned with the calculated one. Note again that the experimental particle charges are slightly smaller than the calculated ones and $\Delta$ used in the calculation is slightly larger than the experimental one (which is in addition not a constant for a given $P_{\mathrm{W}}$ but slightly decreases with $p_{\mathrm{Ar}}$ ). In order to calculate the true MCI pressure threshold, it would be necessary to take into account the evolution of the horizontal confinement as a function of $p_{\mathrm{Ar}}$ and $P_{\mathrm{W}}$ as well as self-consistent wake parameters for the calculation of the lattice modes. A more sophisticated model for the ion wake such as those described in Refs. [78,80,81] is also needed as the wake model has a strong influence on the shape of the lattice modes [78].

To conclude, let us discuss the trend for the crystallization pressure observed in Fig. 4. As was shown in Sec. IV, the crystallization pressure follows the same trend as the MCI threshold pressure: it decreases with increasing power. This can be roughly explained through the modification of the sheath profile. When the $P_{\mathrm{W}}$ increases, the sheath electric field and therefore the vertical confinement become stronger. However, when in the fluid phase, the crossing of the outof-plane mode with the longitudinal in-plane mode always occurs and MCI will exist unless damping due to neutral friction is high enough to suppress it [26]. At constant $\mathrm{rf}$ power, the microparticle charges decrease when increasing pressure (see Fig. 9 and Table I). Therefore, the longitudinal in-plane mode is not as steep (the longitudinal sound speed decreases; see Table I) and it will result in smaller instability growth rate. It was also shown in Ref. [26], in the framework of the wakelayer model, that when the vertical confinement frequency is increased above the threshold for mode crossing for MCI in a 2D crystal with the same microparticle parameters as in the fluid layer (same microparticle charges and same microparticle number density), the instability growth rate decreases as $\sim \exp \left(-\frac{1}{2} \omega_{v}^{2} \delta\right)$. The crystallization threshold is therefore also influenced by the wake parameters. Since these parameters are unknown, the crystallization pressures were not calculated but ion wakes are expected to have a non-negligible effect as for the MCI threshold pressure in 2D complex plasma crystals. A sweep of wake parameter space could be performed to find values that match the best the experimental observations but it is out of scope of the current article. In future studies, the use of a self-consistent wake model such as in Ref. [81] in addition to proper modeling of the horizontal confinement as a function of discharge parameters should enable us to derive self-consistently the MCI threshold and crystallization pressures.

\section{SUMMARY AND CONCLUSION}

In this article, the MCI threshold pressure and crystallization pressure of monolayer complex plasma levitating in the sheath of an argon cc-rf discharge were investigated. It was 
found that the stability of a crystalline microparticle monolayer increases with pressure and with the rf power: at constant rf power, the higher the rf power the lower the crystallization and MCI threshold pressures; at constant background argon pressure, the higher the pressure the lower the rf power for crystallization of the monolayer and for the triggering of MCI. It was also found by measuring the longitudinal and transverse sound speeds in two-dimensional complex plasma crystal that at constant rf power, lower pressure resulted in larger sound speeds and therefore larger microparticle charges. These results are in agreement with previous observations of the MCI $[9,25]$.

A simple rf sheath model was developed to calculate the evolution of the rf sheath profile as a function of the input rf power and the background argon pressure. Using experimental measurements of the electron density in the plasma bulk and the powered electrode self-bias as input parameters, the model was able to show that the argon pressure and the rf power have significant influence on sheath profiles. Using the calculated sheath parameters, the microparticle equilibrium charge, the equilibrium levitation height, and the vertical confinement frequency were also calculated. It was found that (i) a power increase at constant pressure leads to increase of $Z_{d}, z_{\text {lev }}$ and $f_{0_{\mathrm{v}}}$ and (ii) a pressure increase at constant rf power leads to decrease of $Z_{d}, z_{\text {lev }}$ and an increase of $f_{0_{\mathrm{v}}}$. Combined with the point-charge wake model [24], the trends for MCI threshold pressure could be reproduced (i.e., decreasing the pressure at constant power would lead to the crossing of the longitudinal in-plane mode and the out-of-plane mode and trigger MCI, lower rf power results in higher MCI threshold pressure). However, it was also shown that the MCI threshold is sensitive to interparticle distance and wake parameters.

Compared to earlier studies, a better integration of the experimental results and theory was achieved by operating with actual experimental control parameters such as the gas pressure and the discharge power. A sweep of wake parameters space could be performed to find values that match the best experimental observations. However, future studies will concentrate on the use of a self-consistent wake model [81] to study the influence of ion wakes on dust equilibrium position and vertical resonance frequency. The influence of the plasma parameters on horizontal confinement should also be taken into account in order to model self-consistently the interparticle distance in monolayer complex plasma enabling more detailed investigations of fluid MCI and the crystallization threshold pressure.

\section{ACKNOWLEDGMENTS}

The authors would like to thank S. Zhdanov for fruitful comments and discussions. The authors would also like to thank H. Thomas for his careful reading of our manuscript. L.C. acknowledges the support of the Natural Sciences and Engineering Research Council of Canada (NSERC), Grant No. RGPIN-2019-04333.

\section{APPENDIX A: CRYSTALLIZATION OF THE MONOLAYER}

The crystallization process was similar to the one happening during a below MCI threshold localized laser induced melting reported in Ref. [32]. Until the crystallization pressure threshold $p_{\text {cryst }}$ was overcome, the microparticle kinetic energy remained quite large (a few eV). When $p_{\text {cryst }}$ was finally reached, a rapid cooling of the monolayer was induced (in a matter of a few seconds) due to the neutral drag damping rate being higher than the fluid MCI growth rate, and the crystallization occurred (average bond orientational order $\left\langle\Psi_{6}\right\rangle$ increased to a value close to 1 and the numbers of 5 -fold and 7-fold defects decreased tremendously). In this article, the particle kinetics at crystallization threshold was not investigated in detail and was only detected with the naked eye. As illustrated in Fig. 11 for a similar experiment (i.e., different monolayer), the recrystallization event is unmissable.
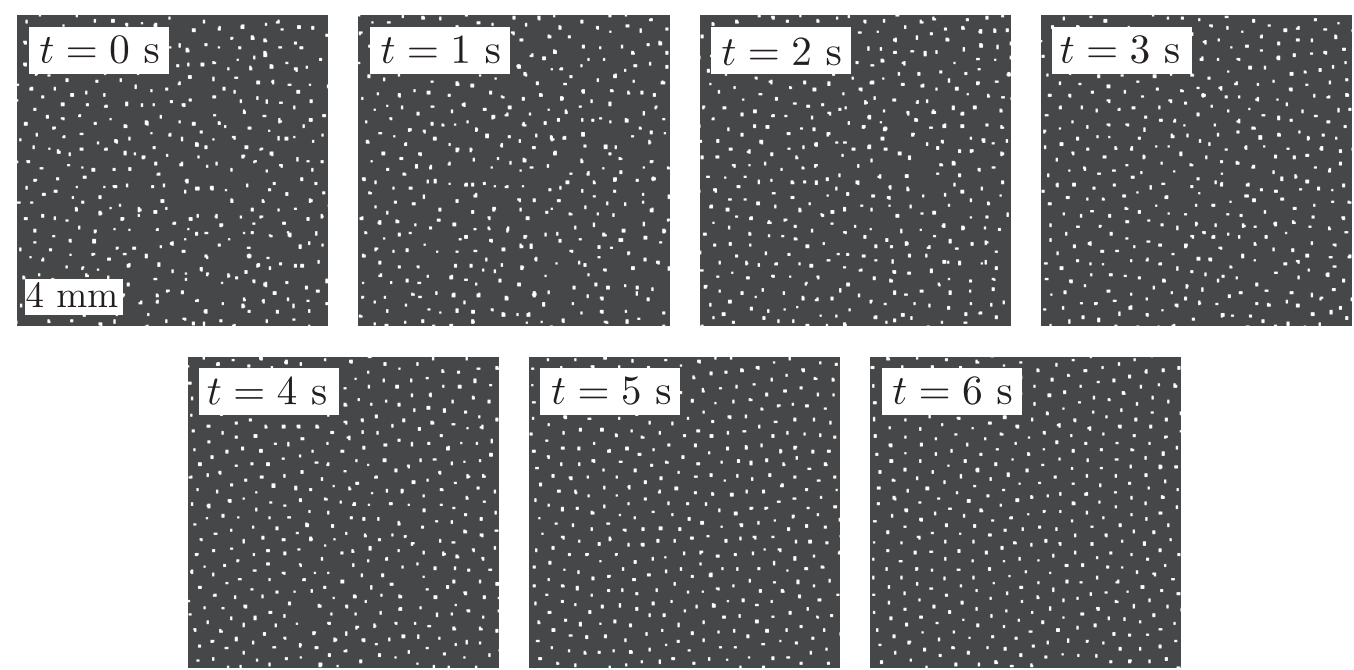

FIG. 11. Snapshots of a microparticle monolayer during recrystallization at $p_{\mathrm{Ar}}=0.90 \mathrm{~Pa}$ and $P_{\mathrm{W}}=5 \mathrm{~W}$. Note that this is a different smaller monolayer than the one used for the results reported in the main text. Therefore the MCI and crystallization thresholds are also different and the above images should only be used as an illustration. 


\section{APPENDIX B: MODE-CROSSING WITHOUT ION WAKE}

The influence of the interparticle distance on mode crossing is investigated in the case where ion wakes are ignored $\left(\sigma_{x, y}=0\right)$. Using the results of Sec. VC and Sec. VIA, the minimum pressure for out-of-plane and in-plane longitudinal mode crossing as a function of power was calculated for different $\Delta(420 \mu \mathrm{m}, 450 \mu \mathrm{m}$, and $500 \mu \mathrm{m})$. The results are presented in Fig. 12. As can be seen, the out-of-plane and in-plane longitudinal mode crossing minimum pressure decreases when increasing the rf power. This can be understood due to the competing effects of the pressure decrease, which makes the sheath longer and therefore weakens the confining electric field, and the rf power increase, which increases the self-bias of the powered electrode and makes the vertical confinement stiffer. Moreover, at low pressure the microparticle charge is larger due to a higher electron temperature, which for a fixed interparticle distance renders the interaction force greater and therefore increases the maximum frequency of the longitudinal in-plane mode and makes mode crossing easier. In addition, one can see that the pressure threshold is systematically lower for larger $\Delta$. This is due to the weaker interparticle interaction which reduces the maximum frequency of the longitudinal in-plane mode and the minimum frequency of the out-of-plane mode. Finally, we can see that experimen-

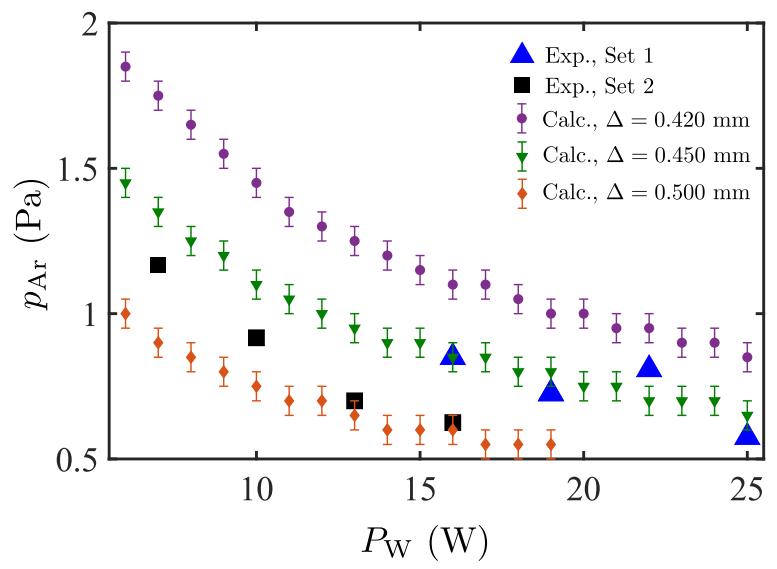

FIG. 12. Pressure at which the crossing of the in-plane longitudinal mode and the out-of-plane mode occurs as a function of rf power for different interparticle distances $\Delta$ and without ion wakes. The experimental MCI pressure thresholds are shown for comparison.

tal points follow roughly the same trend. This shows that the sheath profile (which depends on $p_{\mathrm{Ar}}$ and $P_{\mathrm{W}}$ ) plays a major role in the crossing of the modes and the triggering of $\mathrm{MCI}$ in 2D complex plasma crystals.
[1] G. E. Morfill and H. Thomas, Plasma crystal, J. Vac. Sci. Technol. A 14, 490 (1996).

[2] A. Melzer, S. Nunomura, D. Samsonov, Z. W. Ma, and J. Goree, Laser-excited Mach cones in a dusty plasma crystal, Phys. Rev. E 62, 4162 (2000).

[3] S. Nunomura, D. Samsonov, and J. Goree, Transverse Waves in a Two-Dimensional Screened-Coulomb Crystal (Dusty Plasma), Phys. Rev. Lett. 84, 5141 (2000).

[4] V. A. Schweigert, I. V. Schweigert, V. Nosenko, and J. Goree, Acceleration and orbits of charged particles beneath a monolayer plasma crystal, Phys. Plasmas 9, 4465 (2002).

[5] V. Nosenko, J. Goree, Z. Ma, D. Dubin, and A. Piel, Compressional and shear wakes in a two-dimensional dusty plasma crystal, Phys. Rev. E 68, 056409 (2003).

[6] S. Nunomura, J. Goree, S. Hu, X. Wang, A. Bhattacharjee, and K. Avinash, Phonon Spectrum in a Plasma Crystal, Phys. Rev. Lett. 89, 035001 (2002).

[7] K. Qiao and T. W. Hyde, Dispersion properties of the out-ofplane transverse wave in a two-dimensional Coulomb crystal, Phys. Rev. E 68, 046403 (2003).

[8] L. Couëdel and V. Nosenko, Tracking and linking of microparticle trajectories during mode-coupling induced melting in a two-dimensional complex plasma crystal, J. Imaging 5, 41 (2019).

[9] L. Couëdel, V. Nosenko, A. V. Ivlev, S. K. Zhdanov, H. M. Thomas, and G. E. Morfill, Direct Observation of ModeCoupling Instability in Two-Dimensional Plasma Crystals, Phys. Rev. Lett. 104, 195001 (2010).

[10] D. Samsonov, S. Zhdanov, and G. Morfill, Vertical wave packets observed in a crystallized hexagonal monolayer complex plasma, Phys. Rev. E 71, 026410 (2005).
[11] P. Qiu, T. Sun, and Y. Feng, Observation of the solid and liquid separation after the shock propagation in a two-dimensional Yukawa solid, Phys. Plasmas 28, 113702 (2021).

[12] A. Kananovich and J. Goree, Shock width measured under liquid and solid conditions in a two-dimensional dusty plasma, Phys. Rev. E 104, 055201 (2021).

[13] H. M. Thomas and G. E. Morfill, Melting dynamics of a plasma crystal, Nature (London) 379, 806 (1996).

[14] J. Berumen and J. Goree, Experiment and model for a Stokes layer in a strongly coupled dusty plasma, Phys. Rev. E 104, 035208 (2021).

[15] B. Liu, J. Goree, M. Y. Pustylnik, H. M. Thomas, V. E. Fortov, A. M. Lipaev, A. D. Usachev, O. F. Petrov, A. V. Zobnin, and M. H. Thoma, Time-dependent shear motion in a strongly coupled dusty plasma in PK-4 on the International Space Station (ISS), IEEE Trans. Plasma Sci. 49, 2972 (2021).

[16] E. V. Vasilieva, O. F. Petrov, and M. M. Vasiliev, Laser-induced melting of two-dimensional dusty plasma system in RF discharge, Sci. Rep. 11, 523 (2021).

[17] O. Ishihara and S. V. Vladimirov, Wake potential of a dust grain in a plasma with ion flow, Phys. Plasmas 4, 69 (1997).

[18] A. Melzer, V. A. Schweigert, and A. Piel, Measurement of the Wakefield attraction for "dust plasma molecules", Phys. Scr. 61, 494 (2000).

[19] S. V. Vladimirov, S. A. Maiorov, and O. Ishihara, Molecular dynamics simulation of plasma flow around two stationary dust grains, Phys. Plasmas 10, 3867 (2003).

[20] I. V. Schweigert, V. A. Schweigert, and F. M. Peeters, Perturbation of collisional plasma flow around a charged dust particle: Kinetic analysis, Phys. Plasmas 12, 113501 (2005). 
[21] L. S. Matthews, D. L. Sanford, E. G. Kostadinova, K. S. Ashrafi, E. Guay, and T. W. Hyde, Dust charging in dynamic ion wakes, Phys. Plasmas 27, 023703 (2020).

[22] A. V. Ivlev and G. Morfill, Anisotropic dust lattice modes, Phys. Rev. E 63, 016409 (2000).

[23] A. V. Ivlev, U. Konopka, G. Morfill, and G. Joyce, Melting of monolayer plasma crystals, Phys. Rev. E 68, 026405 (2003).

[24] S. K. Zhdanov, A. V. Ivlev, and G. E. Morfill, Mode-coupling instability of 2D plasma crystals, Phys. Plasmas 16, 083706 (2009).

[25] L. Couëdel, S. K. Zhdanov, A. V. Ivlev, V. Nosenko, H. M. Thomas, and G. E. Morfill, Wave mode coupling due to plasma wakes in two-dimensional plasma crystals: In-depth view, Phys. Plasmas 18, 083707 (2011).

[26] A. V. Ivlev, S. K. Zhdanov, M. Lampe, and G. E. Morfill, Mode-Coupling Instability in a Fluid Two-Dimensional Complex Plasma, Phys. Rev. Lett. 113, 135002 (2014).

[27] S. V. Vladimirov, P. V. Shevchenko, and N. F. Cramer, Vibrational modes in the dust-plasma crystal, Phys. Rev. E 56, R74 (1997).

[28] L. Couëdel, V. Nosenko, S. K. Zhdanov, A. V. Ivlev, H. M. Thomas, and G. E. Morfill, First Direct Measurement of Optical Phonons in 2D Plasma Crystals, Phys. Rev. Lett. 103, 215001 (2009).

[29] S. O. Yurchenko, E. V. Yakovlev, L. Couëdel, N. P. Kryuchkov, A. M. Lipaev, V. N. Naumkin, A. Y. Kislov, P. V. Ovcharov, K. I. Zaytsev, E. V. Vorob'ev, G. E. Morfill, and A. V. Ivlev, Flame propagation in two-dimensional solids: Particle-resolved studies with complex plasmas, Phys. Rev. E 96, 043201 (2017).

[30] J. D. Williams, E. Thomas, L. Couëdel, A. V. Ivlev, S. K. Zhdanov, V. Nosenko, H. M. Thomas, and G. E. Morfill, Kinetics of the melting front in two-dimensional plasma crystals: Complementary analysis with the particle image and particle tracking velocimetries, Phys. Rev. E 86, 046401 (2012).

[31] T. B. Röcker, L. Couëdel, S. K. Zhdanov, V. Nosenko, A. V. Ivlev, H. M. Thomas, and G. E. Morfill, Nonlinear regime of the mode-coupling instability in 2D plasma crystals, Europhys. Lett. 106, 45001 (2014).

[32] L. Couëdel, V. Nosenko, M. Rubin-Zuzic, S. Zhdanov, Y. Elskens, T. Hall, and A. V. Ivlev, Full melting of a twodimensional complex plasma crystal triggered by localized pulsed laser heating, Phys. Rev. E 97, 043206 (2018).

[33] T. B. Röcker, A. V. Ivlev, S. K. Zhdanov, and G. E. Morfill, Effect of strong wakes on waves in two-dimensional plasma crystals, Phys. Rev. E 89, 013104 (2014).

[34] M. A. Lieberman, Analytical solution for capacitive rf sheath, IEEE Trans. Plasma Sci. 16, 638 (1988).

[35] M. A. Lieberman, Dynamics of a collisional, capacitive rf sheath, IEEE Trans. Plasma Sci. 17, 338 (1989).

[36] V. A. Godyak and N. Sternberg, Dynamic model of the electrode sheath in a symmetrically driven plasma, Phys. Rev. A 42, 2299 (1990).

[37] M. Chandhok and J. W. Grizzle, Modeling the pressure dependence of dc bias voltage in asymmetric, capacitive rf sheaths, IEEE Trans. Plasma Sci. 26, 181 (1998).

[38] J. Gierling and K.-U. Riemann, Comparison of a consistent theory of radio frequency sheaths with step models, J. Appl. Phys. 83, 3521 (1998).
[39] M. A. Sobolewski, Sheath model for radio-frequency-biased, high-density plasmas valid for all $\omega / \omega_{i}$, Phys. Rev. E 62, 8540 (2000).

[40] M. N. A. Dewan, P. J. McNally, and P. A. F. Herbert, Plasma modeling for a nonsymmetric capacitive discharge driven by a nonsinusoidal radio frequency current, J. Appl. Phys. 91, 5604 (2002).

[41] P. Chabert and N. Braithwaite, Physics of Radio-Frequency Plasmas (Cambridge University Press, Cambridge, 2011).

[42] U. Czarnetzki, Analytical model for the radio-frequency sheath, Phys. Rev. E 88, 063101 (2013).

[43] P. Chabert, T. V. Tsankov, and U. Czarnetzki, Foundations of capacitive and inductive radio-frequency discharges, Plasma Sources Sci. Technol. 30, 024001 (2021).

[44] V. Nosenko, A. V. Ivlev, S. K. Zhdanov, M. Fink, and G. E. Morfill, Rotating electric fields in complex (dusty) plasmas, Phys. Plasmas 16, 083708 (2009).

[45] V. Nosenko, J. Goree, and A. Piel, Laser method of heating monolayer dusty plasmas, Phys. Plasmas 13, 032106 (2006).

[46] V. Nosenko, S. Zhdanov, and G. Morfill, Supersonic Dislocations Observed in a Plasma Crystal, Phys. Rev. Lett. 99, 025002 (2007).

[47] M. A. Lieberman and A. J. Lichtenberg, Principles of Plasma Discharges and Materials Processing (Wiley, New York, 1994).

[48] Y. P. Raizer, M. N. Shneider, and N. A. Yatsenko, RadioFrequency Capacitive Discharges (CRC Press, Boca Raton, FL, 1995).

[49] Y. P. Song, D. Field, and D. F. Klemperer, Electrical potentials in RF discharges, J. Phys. D 23, 673 (1990).

[50] V. Land, E. Shen, B. Smith, L. Matthews, and T. Hyde, Experimental and computational characterization of a modified GEC cell for dusty plasma experiments, New J. Phys. 11, 063024 (2009).

[51] N. Kohlmann, F. Wieben, O. H. Asnaz, D. Block, and F. Greiner, High-precision in situ size measurements of single microparticles in an rf plasma, Phys. Plasmas 26, 053701 (2019).

[52] Except when within a few $10^{-2} \mathrm{~Pa}$ of the crystallization pressure where the outer part of the monolayer has started crystallizing while the center remained melted.

[53] L. Couëdel, S. Zhdanov, V. Nosenko, A. V. Ivlev, H. M. Thomas, and G. E. Morfill, Synchronization of particle motion induced by mode coupling in a two-dimensional plasma crystal, Phys. Rev. E 89, 053108 (2014).

[54] I. Laut, C. Räth, S. Zhdanov, V. Nosenko, L. Couëdel, and H. M. Thomas, Synchronization of particle motion in compressed two-dimensional plasma crystals, Europhys. Lett. 110, 65001 (2015).

[55] The pressure was kept constant during imaging.

[56] J.-P. Hansen and I. R. McDonald, Theory of Simple Liquids, 3rd ed. (Elsevier/Academic Press, Cambridge, 2006).

[57] B. Liu, J. Goree, V. E. Fortov, A. M. Lipaev, V. I. Molotkov, O. F. Petrov, G. E. Morfill, H. M. Thomas, H. Rothermel, and A. V. Ivlev, Transverse oscillations in a single-layer dusty plasma under microgravity, Phys. Plasmas 16, 083703 (2009).

[58] N. P. Kryuchkov, L. A. Mistryukova, V. V. Brazhkin, and S. O. Yurchenko, Excitation spectra in fluids: How to analyze them properly, Sci. Rep. 9, 10483 (2019).

[59] S. Khrapak and L. Couëdel, Dispersion relations of Yukawa fluids at weak and moderate coupling, Phys. Rev. E 102, 033207 (2020). 
[60] D. H. E. Dubin, The phonon wake behind a charge moving relative to a two-dimensional plasma crystal, Phys. Plasmas 7, 3895 (2000).

[61] S. Nunomura, J. Goree, S. Hu, X. Wang, and A. Bhattacharjee, Dispersion relations of longitudinal and transverse waves in two-dimensional screened Coulomb crystals, Phys. Rev. E 65, 066402 (2002).

[62] P. Hartmann, G. J. Kalman, Z. Donkó, and K. Kutasi, Equilibrium properties and phase diagram of two-dimensional Yukawa systems, Phys. Rev. E 72, 026409 (2005).

[63] V. A. Godyak and N. Sternberg, Smooth plasma-sheath transition in a hydrodynamic model, IEEE Trans. Plasma Sci. 18, 159 (1990).

[64] M. Moisan and J. Pelletier, Physique des Plasmas Collisionnels: Application aux Décharges Haute Fréquence (Grenoble Sciences, Bonchamp-Lès-Laval, 2006).

[65] J. Schulze, B. G. Heil, D. Luggenhölscher, R. P. Brinkmann, and U. Czarnetzki, Stochastic heating in asymmetric capacitively coupled RF discharges, J. Phys. D 41, 195212 (2008).

[66] M. L. Hause, B. D. Prince, and R. J. Bemish, Krypton charge exchange cross sections for Hall effect thruster models, J. Appl. Phys. 113, 163301 (2013).

[67] A. V. Zobnin, A. D. Usachev, O. F. Petrov, and V. E. Fortov, Ion current on a small spherical attractive probe in a weakly ionized plasma with ion-neutral collisions (kinetic approach), Phys. Plasmas 15, 043705 (2008).

[68] S. Khrapak and G. Morfill, Basic processes in complex (dusty) plasmas: Charging, interactions, and ion drag force, Contrib. Plasma Phys. 49, 148 (2009).

[69] T. Trottenberg, A. Melzer, and A. Piel, Measurement of the electric charge on particulates forming Coulomb crystals in the sheath of a radiofrequency plasma, Plasma Sources Sci. Technol. 4, 450 (1995).

[70] A. Homann, A. Melzer, and A. Piel, Measuring the charge on single particles by laser-excited resonances in plasma crystals, Phys. Rev. E 59, R3835 (1999).
[71] E. B. Tomme, D. A. Law, B. M. Annaratone, and J. E. Allen, Parabolic Plasma Sheath Potentials and Their Implications for the Charge on Levitated Dust Particles, Phys. Rev. Lett. 85, 2518 (2000).

[72] A. Melzer, Laser manipulation of particles in dusty plasmas, Plasma Sources Sci. Technol. 10, 303 (2001).

[73] B. J. Harris, L. S. Matthews, and T. W. Hyde, Vertical-probeinduced asymmetric dust oscillation in complex plasma, Phys. Rev. E 87, 053109 (2013).

[74] S. K. Zhdanov, L. Couëdel, V. Nosenko, H. M. Thomas, and G. E. Morfill, Spontaneous pairing and cooperative movements of micro-particles in a two dimensional plasma crystal, Phys. Plasmas 22, 053703 (2015).

[75] M. Jambor, V. Nosenko, S. K. Zhdanov, and H. M. Thomas, Plasma crystal dynamics measured with a three-dimensional plenoptic camera, Rev. Sci. Instrum. 87, 033505 (2016).

[76] V. A. Schweigert, I. V. Schweigert, A. Melzer, A. Homann, and A. Piel, Alignment and instability of dust crystals in plasmas, Phys. Rev. E 54, 4155 (1996).

[77] A. Melzer, V. A. Schweigert, I. V. Schweigert, A. Homann, S. Peters, and A. Piel, Structure and stability of the plasma crystal, Phys. Rev. E 54, R46 (1996).

[78] T. B. Röcker, A. V. Ivlev, R. Kompaneets, and G. E. Morfill, Mode coupling in two-dimensional plasma crystals: Role of the wake model, Phys. Plasmas 19, 033708 (2012).

[79] J. K. Meyer, I. Laut, S. K. Zhdanov, V. Nosenko, and H. M. Thomas, Coupling of Noncrossing Wave Modes in a TwoDimensional Plasma Crystal, Phys. Rev. Lett. 119, 255001 (2017).

[80] T. B. Röcker, S. K. Zhdanov, A. V. Ivlev, M. Lampe, G. Joyce, and G. E. Morfill, Effective dipole moment for the mode coupling instability: Mapping of self-consistent wake models, Phys. Plasmas 19, 073708 (2012).

[81] R. Kompaneets, G. E. Morfill, and A. V. Ivlev, Wakes in complex plasmas: A self-consistent kinetic theory, Phys. Rev. E 93, 063201 (2016). 